\title{
Incorporating Uncertainty into Project Schedule Crashing:
}

\section{An Algorithm}

\author{
E.S. Subhy ${ }^{a}$, M.E. Georgy ${ }^{b}$ and M.E. Ibrahim ${ }^{c}$ \\ ${ }^{a}$ Faculty of Engineering, Fayoum University, Egypt \\ ${ }^{b}$ School of Property, Construction and Project Management, RMIT University, Australia \\ ${ }^{c}$ Faculty of Engineering, Cairo University, Egypt \\ E-mail: ess1@fayoum.edu.eg, maged.georgy@rmit.edu.au,moheeb.elsaid@eng.cu.edu.eg
}

\begin{abstract}
-
The uncertain environment in which construction projects are executed poses a challenge to project managers and planners alike as they go about planning and controlling these endeavours. Statistical and simulation models have been devised over the years to assist in estimating the time and cost of construction activities while accounting for uncertainty. However, most of these models fail to comprehensively associate these uncertainty-mindful estimates with the varied resource patterns/configurations that may undertake the work. As a result, when performing schedule crashing, one could end up with a very different strategy for project execution when accounting for all uncertainties compared to the deterministic counterpart. As such this paper presents a dynamic simulation algorithm for project schedule crashing. The devised algorithm incorporates computer simulation into the resource-time-cost triad at the activity and project levels. To perform the dynamic simulation, successive schedule simulations are created with each corresponding to a particular resource pattern/configuration that could possibly be used for executing project activities. After each simulation run is completed, parameters such as the project's completion time and direct cost are estimated against a certain degree of confidence. The combination of resource patterns for project activities that delivers the minimum project cost is then utilized to produce the optimal-cost project schedule. The computer-automated algorithm is exemplified via a simple project scenario. Results are compared to the traditional approach for least cost scheduling, which show how ignoring the uncertainty dimension could result in strategies far from being optimal.
\end{abstract}

Keywords -

Project scheduling; Crashing; Construction resources; Simulation; Optimum construction cost

\section{Introduction}

The reliable estimate of resources, durations and costs for the various project activities is fundamental to its successful planning and control. The deterministic representation of such parameters, also known as singlepoint estimating, is the norm in developing project schedules. However, the environment in which construction projects are executed is quite dynamic. A plethora of events can influence the construction work, whether positively or negatively. And hence, the confidence in the deterministic estimates is occasionally questioned.

A number of researchers attempted to depict the project parameters stochastically. In this context, multiple values are considered for each parameter into consideration. Probability distributions are utilized to signify the chance each value can occur in reality. With such representation, computer simulation can follow [1] to simulate the project behavior and the many scenarios that can possibly occur.

The stochastic simulation, classified as either discrete-event or continuous, is defined as:

"a technique to make prediction of system performance and to understand its behavior" [2].

Generally, Monte Carlo simulation is the more commonly used platform for the stochastic scheduling and costing in projects. In construction, specific simulators have been devised over the years such as CYCLONE [3], STROPOSCOPE [4], PICASSO [5], among others.

\section{Research Need and Approach}

In the deterministic analysis, concepts of schedule crashing are normally applied to satisfy the project's requirements and completion date constraints. Such concepts are sometimes referred to by the term least- 
cost scheduling as they aim to arrive at the least possible cost that satisfies the project's requirements and time constraint. The literature is quite rich in discussing these concepts based on the deterministic representation of resources, durations, and costs $[6,7,8]$.

Realizing the importance of stochastic modeling and due to the lack of researches into stochastic schedule crashing, a study was initiated by the authors.

The paper at hand presents a new schedule planning and updating technique, referred to as the Dynamic Simulation for Optimal-Cost Scheduling. Technique establishes comprehensive algorithms for both project planning and the more sophisticated schedule updating. Paper particularly focuses on project planning and introduces the algorithm used for such purpose. It explains how to account for the various resource patterns/configurations used for job execution. Finally, a comparison of the typical least-cost scheduling process and the dynamic simulation approach is presented via an example construction project.

\section{Modeling the Resource-Duration Relationship}

The productive resources assigned to a construction activity determine the cost and length of time it takes to complete it. As known, the more resources allocated to a job, the less duration and more cost it has. When accounting for the uncertainty in work execution, a given resource configuration/pattern will cause an activity to be executed in a duration that possibly ranges from a low/minimum/optimistic value $(o)$ to a high/maximum/pessimistic value $(p)$. Within this range, a most likely estimate $(m)$ corresponds to the duration having the highest probability of occurrence amongst all values possible.

Let us assume the minimum and maximum levels of productive resources to be $R_{\min }$ and $R_{\max }$, which correspond to durations $D_{\min }(p, m, o)$ and $D_{\max }(p, m, o)$. A triangular distribution is the distribution of choice in this study. The unavailability of abundant data in construction companies makes this distribution a more suitable choice in real-world practice. Further, its usage has been promoted in the process simulation literature $[9,10]$. With the triangular distribution in place, the relationship between the resource usage and activity duration can be represented in the $3 \mathrm{D}$ view illustrated in figure 1. The human resource order refers to the level/number of human resources assigned to the construction job. The least order of human resources corresponds to the longest duration and vice versa. Given the manpower wage rates, the human resource order can be converted into dollar values.

The triangular probability distribution can be converted into the cumulative probability distribution illustrated in figure 2. This distribution is fundamental to the randomization process and the choice of resource patterns and duration values for the subsequent simulation runs.

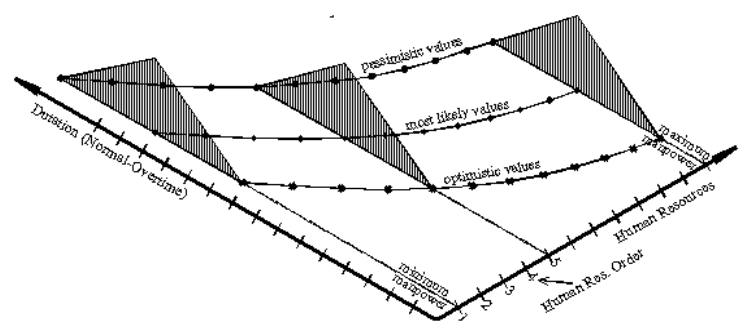

Figure 1. Resource-duration relationship (case of unlimited resources)

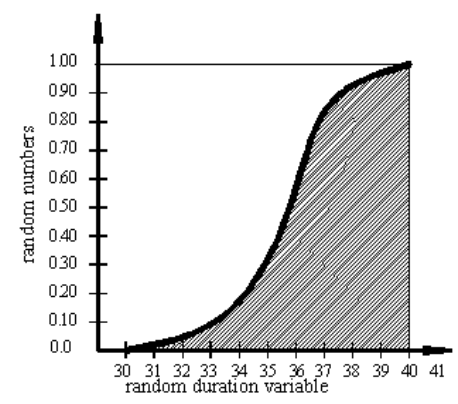

Figure 2. Cumulative probability distribution (case of unlimited resources)

The representation in figures 1 and 2 assumes no limitation on the productive resources and/or materials used for work execution. However, in reality, some activities in the project will typically have such limitations, figures 3 and 4 . As seen, the limitation imposes a minimum possible duration of $C_{i}$ on activity $i$. Consider for instance insufficient materials or construction tools that prevents completing the work in less than $C_{i}$. Even if the manpower assigned to work can complete the work in less time, the possibility is eliminated.

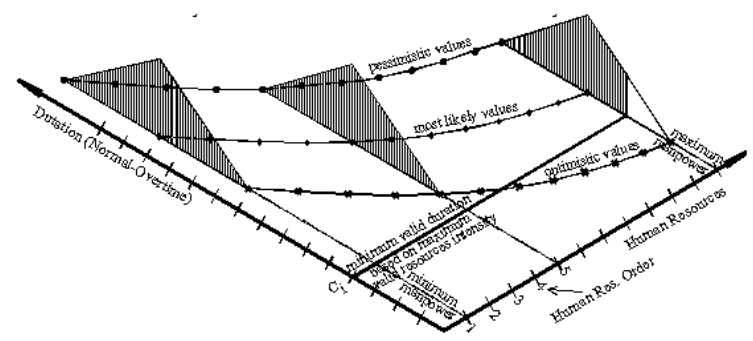

Figure 3. Resource-duration relationship (case of limited resources) 


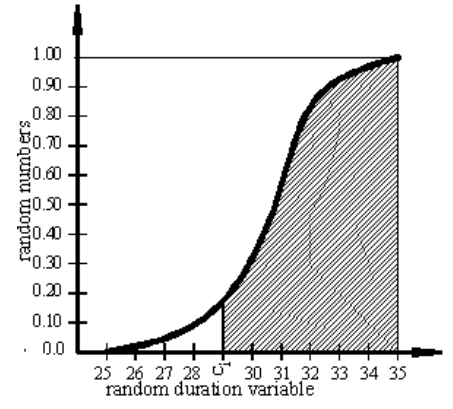

Figure 4. Cumulative probability distribution (case of limited resources)

\section{Algorithm for Simulating the Project Schedule and the Crashing Process}

A project's activities need to be executed in a certain order so as to deliver the project on time and on budget. If a certain set of resource configurations/patterns are selected for the various project activities, computer simulation can then be utilized to estimate the possible completion times for such project. Typically a probability distribution for the total project duration is developed. Using this distribution, the probability of completion by a certain date is estimated.

In a sense, this stochastic analysis determines how risky the entire project is, with regard to its completion by a pre-set date. Also, the corresponding project cost can stochastically be estimated for the selected resource configurations/patterns. Time and cost in this regard represent the performance criteria that govern the development and approval of project plans.

When one examines the process of schedule crashing in case of stochastic analysis, it becomes obvious that the process is rather sophisticated. Simulation turns into a successive process, whose parameters change with the change of resource patterns, activities, critical path(s) in the project network, etc. To address such an aspect, a strategy has been established in this research to approach the crashing process.

The stochastic analysis starts with the so-called "Base Simulation". Minimum productive resources (maximum duration) are assigned to each activity as shown in figure 5. This simulation run is denoted with $\operatorname{Sim}_{0}$.

Using the minimum-resource probability distributions for all project activities, the project's probabilistic time and cost are developed. These cumulative probability charts provide the basis for moving to the next step. It is understandable that each subsequent simulation, i.e., $\operatorname{Sim}_{1}, \mathrm{Sim}_{2}$, etc., will associate with a different resource pattern. Results of the various steps are organized in vectors and matrices as shown in figure 6.

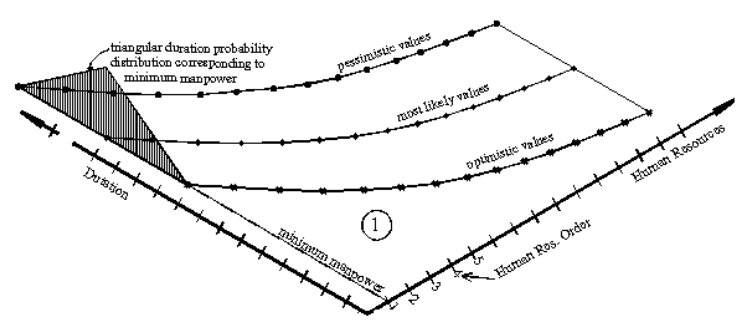

Figure 5. Base Simulation - minimum resource configuration

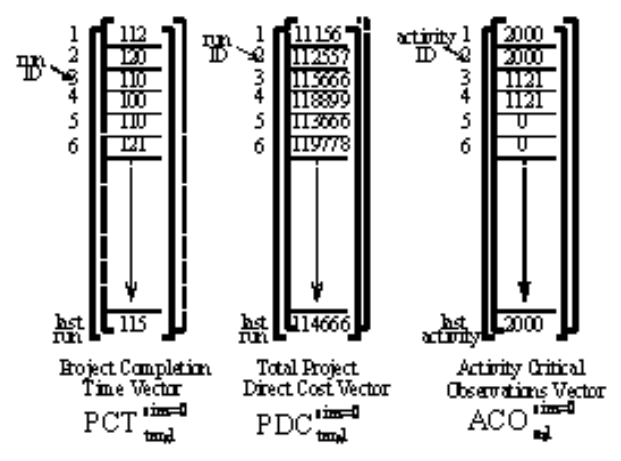

Figure 6. Simulation results recording

The third vector in figure 6 is particularly important as it tracks the criticality of each activity in the project (i.e., the number of times the activity was recorded as critical in a given simulation). This guides the immediately subsequent simulation of the project and which activities to shorten.

The dynamic simulation process proceeds as per the following steps:

1. Activities not utilizing the maximum possible resource configuration, i.e., there is a window to assign more resources to reduce the duration of the activity, are identified.

2. A preferential analysis is performed to select activities worthy of being assigned more resources in the crashing process. This preferential analysis is designed to take into account four aspects; they are:

- Utilizing the information of the preceding simulation to progress forward;

- Observing the criticality of all activities having potential for crashing;

- Observing the cost increase per unit time for each of the above activities; and 
- Observing the multiplicity and parallelism in critical paths.

In conclusion, for any valid-for-reduction activity $i$, the expected increase in the project direct cost per one unit reduction in the project completion time, $E C I_{i, \operatorname{sim}(j) \rightarrow \operatorname{sim}(j+1)}$, can be calculated as follows:

$E C I_{i, \operatorname{sim}(j) \rightarrow \operatorname{sim}(j+1)}=\left(\left(D C_{i, \operatorname{sim}(j+1)}-D C_{\operatorname{sim}(j)}\right)\right)$

$\left(\left(D U R_{s i m(j)}-D U R_{i, \operatorname{sim}(j+1)}\right)\right.$

Where $D C_{\text {sim(j) }}$ is the direct project cost at $\operatorname{sim} j$, $D C_{i, s i m(j+1)}$ is the expected direct project cost at sim $j+1$ resulting from manipulating activity $i$, $D U R_{\operatorname{sim}(j)}$ is the project duration at $\operatorname{sim} j$, and $D U R_{i, s i m(j+1)}$ is the expected project duration at sim $j+1$ resulting from manipulating activity $i$. Both $D C_{i, \operatorname{sim}(j+1)}$ and $D U R_{i, \operatorname{sim}(j+1)}$ result from the preferential analysis cited above. Apparently, the minimum $E C I_{i, \operatorname{sim}(j) \rightarrow \operatorname{sim}(j+1)}$ will point out which activity whose duration should be reduced.

3. The activity selected via preferential analysis will be allocated the additional resources needed for the project's time reduction. A new CPM-based simulation, according to the new configuration of resources, is performed to obtain the values of $D U R_{\text {sim }(j)}$ and $D C_{\text {sim }(j)}$ for the subsequent step of the simulation process.

4. The new value $D U R_{\text {sim(j) }}$ is evaluated to judge whether further actions are needed to meet the project's time constraints. If the deadline for project completion is met, the simulation process stops at this stage and the resource patterns/configurations utilized in the last simulation are used in the project plan.

\section{Illustrative Example: Least-Cost Scheduling vs. Dynamic Simulation}

A simple construction project is used to exemplify the presented algorithm and compare with the more traditional least cost scheduling. The project comprises the fabrication of steel template with gratings, where unlimited resources exist. The activities of this project are (1) preparation of shop drawings and materials takeoff; (2) delivery of materials; (3) issuance of shop drawings for fabrication; (4) cutting of template slots; (5) clearing of template slots; (6) rolling of template slots; (7) cutting of gratings; (8) clearing of gratings; (9) rolling of gratings; (10) painting of template slot; (11) galvanization of gratings; (12) inspection; and (13) delivery to site.

\subsection{Least-Cost Scheduling}

Performing a least-cost scheduling exercise for the project results in the values illustrated in table 1 . It is to be noted that the same action is consecutively taken to reduce project duration from 45 to 42 days. Each day in this reduction adds $\$ 120$ per day. This accumulates into $\$ 360$, i.e. 3 times $\$ 120$, when accounting for the 3-day duration reduction.

Table 1 Results of the least cost scheduling application

\begin{tabular}{cccc}
\hline $\begin{array}{c}\text { Days } \\
\text { Shortened }\end{array}$ & $\begin{array}{c}\text { Project } \\
\text { Duration }\end{array}$ & Direct Cost & $\begin{array}{c}\text { Cost } \\
\text { Increase }\end{array}$ \\
\hline 0 & 47 & $\$ 36,355$ & 0 \\
1 & 46 & $\$ 36,415$ & $\$ 60$ \\
2 & 45 & $\$ 36,505$ & $\$ 90$ \\
5 & 42 & $\$ 36,865$ & $\$ 120 \times 3$ \\
6 & 41 & $\$ 37,065$ & $\$ 200$ \\
7 & 40 & $\$ 37,285$ & $\$ 220$ \\
8 & 39 & $\$ 37,585$ & $\$ 300$ \\
9 & 38 & $\$ 37,905$ & $\$ 320$ \\
10 & 37 & $\$ 38,428$ & $\$ 523$ \\
11 & 36 & $\$ 39,356$ & $\$ 928$ \\
12 & 35 & $\$ 40,411$ & $\$ 1,055$ \\
\hline
\end{tabular}

\subsection{Dynamic Simulation Scheduling}

Using the same information, the project was input into a computerized Dynamic Simulation system developed based on the principles and algorithms presented in the paper. First, minimum resources for project activities are assigned, i.e., establishing the basis for running the Base Simulation where days shortened is equal to 0 . Project was simulated using 750 runs, and the results of project schedule are illustrated in figures 7 . Figure 7 shows that a 54.55 -day project schedule has a degree of confidence of $85 \%$ (or a $15 \%$ chance of being exceeded). When compared with the results obtained from the deterministic least-cost scheduling previously performed, a 47-day project schedule (obtained when days shortened $=0$ ) has a degree of confidence of $12 \%$ (or an $88 \%$ chance of being exceeded). Thus, based on the stochastic duration output, a 47-day project schedule provides the contractor with only a $12 \%$ chance that the project can be completed using this schedule duration and resource configuration.

Following to the analysis of the base simulation, the computerized Dynamic Simulation system was used to shorten the project by 1 to 12 days. Analysis of simulation results was performed for each shortened day. Results of the Dynamic Simulation are summarized in table 2 . 


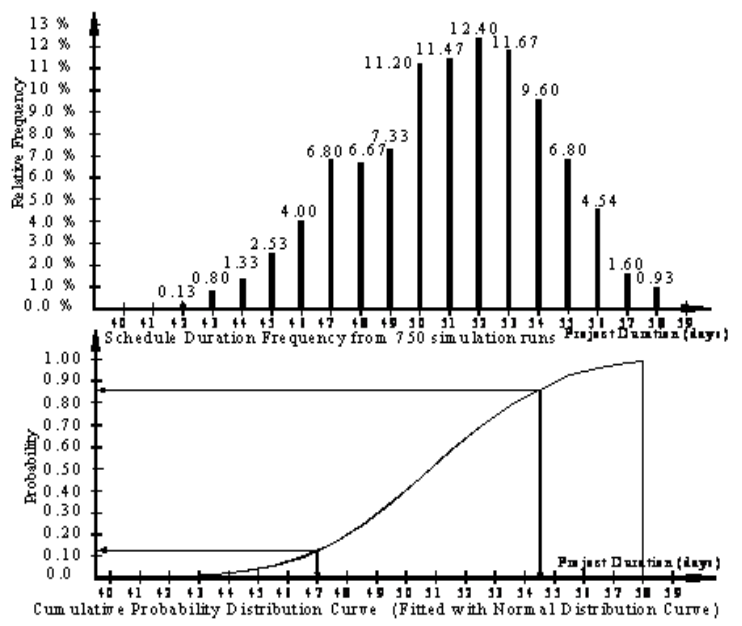

Figure 7. Results for the base simulation

Table 2 Results of the dynamic simulation application

\begin{tabular}{cccc}
\hline $\begin{array}{c}\text { Days } \\
\text { Shortened }\end{array}$ & $\begin{array}{c}\text { Project } \\
\text { Duration }\end{array}$ & $\begin{array}{c}\text { Probability } \\
\text { i.e. degree } \\
\text { of } \\
\text { confidence) }\end{array}$ & $\begin{array}{c}\text { Project } \\
\text { Duration } \\
\text { at } 85 \%\end{array}$ \\
\hline 0 & 47 & $12 \%$ & 54.55 \\
1 & 46 & $11 \%$ & 53.55 \\
2 & 45 & $12 \%$ & 52.55 \\
5 & 42 & $11 \%$ & 49.41 \\
6 & 41 & $12 \%$ & 48.41 \\
7 & 40 & $8 \%$ & 47.50 \\
8 & 39 & $8 \%$ & 46.50 \\
9 & 38 & $8 \%$ & 45.27 \\
10 & 37 & $8 \%$ & 43.80 \\
11 & 36 & $5 \%$ & 43.27 \\
12 & 35 & $4 \%$ & 42.53 \\
\hline
\end{tabular}

\subsection{Comparison of Results}

When comparing the detailed results of the least cost scheduling and dynamic simulation exercises, it becomes apparent that the activities addressed in the crashing process were the same less the sequence. One or more activities, when using the dynamic simulation, may require such a crashing action earlier or later than the traditional approach.

Comparison of the estimated project durations between the two approaches, as illustrated in table 2, shows great diversity in terms chances/probabilities. Values depicted by the traditional approach have quite a low probability of occurrence with the assigned resources and strategies for crashing adopted. When accounting for the variability in resources, durations and costs, the optimum values dramatically change.
While the deterministic least-cost scheduling technique was shown to produce less than satisfactory estimates, it still provides a reasonable guideline for the crashing process steps. The same set of activities was selected though the sequence differed between the two approaches. However, in conclusion, the dynamic simulation approach provides a more comprehensive view of the crashing process that is hard, even impossible, to visualize using the traditional approach.

The reader may have noticed that the analysis have not explicitly addressed indirect costs. Overheads in construction projects are without doubt a fundamental element of the cost. However, the indirect costs are typically tied to and considered a function of project duration [11]. Since both the least cost and dynamic simulation scheduling algorithms follow a step-by-step process for project duration reduction, the indirect costs for each step will be the same for both algorithms. In other words, the indirect cost will not create a different answer when comparing the two algorithms. However, it is understandable when the optimum total project cost is pursued -which is not the focus of this particular paper- incorporating the indirect costs becomes a necessity.

\section{Conclusion}

The actual times and costs for the constituent activities of a project cannot be determined until these activities are carried out in reality. When performing the project planning function, the deterministic techniques are simply lacking. They consider one possible time/cost value for each activity. And as such, they fail to portray the big picture during the planning phase.

A new stochastic technique is developed to better identify the activities that should be shortened in the schedule crashing process (or schedule relaxation if the opposite is sought). This new technique, referred to by the term Dynamic Simulation, can be used in project planning as well as project control. Both cases of unlimited and limited resources are accounted for. This paper focused on the algorithm used for planning purposes.

The proposed algorithm performs successive discrete-event simulations using multiple configurations of resources and their corresponding duration and cost estimates. Each simulation run, with a unique resource configuration, provides a basis for the progressive crashing processes. At the end, the Dynamic Simulation algorithm not only arrives at an optimally-crashed schedule but also provides the optimum level of resources needed to achieve this goal.

The example project demonstrated that less informative decisions can be made when accounting for the deterministic results alone. The dynamic simulation 
approach stochastically accounts for the different resource patterns, job completion times, and the associated costs. The final decision on how to perform project crashing and whether it is possible to complete project by a certain date becomes handy when the latter approach is adopted.

Finally, the paper utilized a triangular distribution for the illustration of the devised algorithm. The adoption of triangular distributions was due to their practicality. However the dynamic simulation algorithm can function with any distribution of choice e.g. uniform, normal, beta, etc. It comes down to which distribution is more appropriate in a given construction application.

\section{References}

[1] Shi J. Computer simulation in AEC and its future development. In Berkeley-Stanford CE\&M Workshop, Stanford, USA, 1999.

[2] Law A. and Kelton W. Simulation Modeling and Analysis, McGraw-Hill, New York, USA, 1991.

[3] Halpin D. and Riggs L. Planning and Analysis of Construction Operations, John Wiley \& Sons, New York, USA, 1992.

[4] Martinez J. STROBOSCOPE: state and resource based simulation of construction processes. Ph.D. Dissertation, University of Michigan, Ann Arbor, USA, 1996.

[5] Senior B. and Halpin D. Simplified simulation system for construction projects. Construction Engineering and Management, 124(1): 72-81, 1998.

[6] Yau C. Project compression: a method for speeding up resource constrained projects which preserve the activity schedule. European Journal of Operational Research, 49(1): 140-152, 1990.

[7] $\mathrm{Wu} \mathrm{H}$. and Li R. Methodology for schedule compression. Production Planning and Control, 7(4): 407-417, 1996.

[8] Al-Tabtabai H. and Alex P. Network compression using genetic algorithms. In Proceedings of the 1998 International Computing Congress on Computing in Civil Engineering, Boston, USA, 1998.

[9] Newton S. Methods of analysing risk exposure in the cost estimates of high quality offices. Construction Management and Economics, 10:431-449, 1992.

[10] Raftery J. Principles of Building Economics, Blackwell Science, Oxford, UK, 1991.
[11] Ahuja H. Dozzi S. and AbouRizk S. Project Management: Techniques in Planning and Controlling Construction Projects, $2^{\text {nd }}$ Edition, John Wiley \& Sons, New York, USA, 1994. 